\title{
Entwicklung von Hochleistungsaerogelbeton
}

\begin{tabular}{|r|l|}
\hline Journal: & Beton- und Stahlbetonbau \\
\hline Manuskript ID & Entwurf \\
\hline Wiley - Manuscript type: & Fachthema \\
\hline Eingangsdatum durch den & n/a \\
\hline Vollständige Liste der Autoren: & $\begin{array}{l}\text { Schnellenbach-Held, Martina; Universitat Duisburg-Essen Fakultat fur } \\
\text { Ingenieurwissenschaften, Institut für Massivbau } \\
\text { Welsch, Torsten; Universitat Duisburg-Essen Fakultat fur } \\
\text { Ingenieurwissenschaften, Institut für Massivbau } \\
\text { Fickler, Silvia; Universitat Duisburg-Essen Fakultat fur } \\
\text { Ingenieurwissenschaften, Institut für Massivbau } \\
\text { Milow, Barbara; Deutsches Zentrum fur Luft und Raumfahrt, Institut für } \\
\text { Werkstoff-Forschung } \\
\text { Ratke, Lorenz; Deutsches Zentrum fur Luft und Raumfahrt, Institut für } \\
\text { Werkstoff-Forschung }\end{array}$ \\
\hline Keywords: & $\begin{array}{l}\text { Aerogelbeton, Leichtbeton, Hochleistungsbeton (HPC), Ultra- } \\
\text { Wochleistungsbeton (UHPC), Gradierter Beton, GFK-Bewehrung, Aerogel, } \\
\text { Wärmedämmung, Wärmeleitfähigkeit }\end{array}$ \\
\hline
\end{tabular}




\section{Entwicklung von Hochleistungsaerogelbeton \\ Development of High Performance Aerogel Concrete}

Martina Schnellenbach-Held, Torsten Welsch, Silvia Fickler, Barbara Milow, Lorenz Ratke

Aufgrund der Anforderungen an den Wärmeschutz ist die Ausführung einschaliger Außenwände aus Normalbeton bereits seit einigen Jahrzehnten nicht mehr praktikabel. Um den bauphysikalischen Anforderungen gerecht zu werden, sind in der Regel zusätzliche Maßnahmen wie die Anbringung von Wärmedämmverbundsystemen oder die Ausführung als zweischalige Wandkonstruktion mit Kerndämmung erforderlich, aus denen gestalterische, konstruktive oder wirtschaftliche Nachteile resultieren können. Bisherige Versuche, einschalige Außenwände aus Leichtbetonen herzustellen, erfordern wegen der verhältnismäßig geringen Druckfestigkeiten oder der im Vergleich zu anderen Außenwandkonstruktionen noch immer höheren Wärmeleitfähigkeiten stets sehr große Wanddicken. Das Institut für Massivbau (IfM) der Universität Duisburg-Essen hat in Zusammenarbeit mit der Abteilung für Werkstoff-Forschung des Deutschen Zentrums für Luft und Raumfahrt (DLR) durch Einbettung von Quarzglas-Aerogelgranulat in UHPC-Matrizen einen Hochleistungsaerogelbeton entwickelt, der ein sehr günstiges Verhältnis zwischen Druckfestigkeit und Wärmeleitfähigkeit aufweist [1]. Nachfolgend werden die mechanischen und bauphysikalischen Eigenschaften des neuen Werkstoffs vorgestellt.

Stichwörter: Aerogelbeton, Leichtbeton, Hochleistungsbeton (HPC), Ultra-Hochleistungsbeton (UHPC), Gradierter Beton, GFK-Bewehrung, Aerogel, Wärmedämmung, Wärmeleitfähigkeit

Because of requirements for thermal insulation the realization of single-leaf walls made of normal concrete is not feasible for a couple of decades. Thus, additional means such as external thermal insulation systems or cavity walls with core insulation are normally necessary, which are accompanied by crea- 
tive, constructive or economical disadvantages. Previous attempts to produce single-leaf walls with lightweight concretes lead to huge wall thicknesses due to the low compressive strength or the higher thermal conductivities of lightweight concretes. By embedding silica aerogel granules in a high strength cement matrix, the Institute for Structural Concrete (ISC) at the University of Duisburg-Essen developed a High Performance Aerogel Concrete (HPAC) in cooperation with the Institute of Materials Research of the German Aerospace Center (DLR). The HPAC exhibits a remarkable relation between compressive strength and thermal conductivity [1]. The following report covers the mechanical and the building physics properties of the new material.

Keywords: Aerogel Concrete, Lightweight Aggregate Concrete, High Performance Concrete (HPC), Ultra High Performance Concrete (UHPC), Graduated Concrete, GFRP Reinforcement, Aerogel, Heat Insulation, Thermal Conductivity

\section{Veranlassung und Zielsetzung}

Einschalige Außenwandkonstruktionen müssen sowohl den Anforderungen an die Tragfähigkeit als auch an den Schall- und Wärmeschutz genügen. Aus statischen Gründen ist eine möglichst hohe Druckfestigkeit notwendig, während in bauphysikalischer Hinsicht eine niedrige Wärmeleitfähigkeit wünschenswert ist. Beide Eigenschaften sind abhängig von der Rohdichte, korrelieren aber negativ. Stahlbeton erfüllt die Anforderungen an die Tragfähigkeit, weist aufgrund seiner hohen Rohdichte von $2400 \mathrm{~kg} / \mathrm{m}^{3}$ aber eine sehr hohe Wärmeleitfähigkeit von $\lambda \approx 2,5 \mathrm{~W} /(\mathrm{mK})$ auf. Einschalige Außenwände aus Stahlbeton können daher nicht ohne weitere Maßnahmen, z.B. die Aufbringung eines Wärmedämmverbundsystems (WDVS) oder die Ausführung als zweischalige Wand mit Kerndämmung, hergestellt werden. Um die hiermit verbundenen gestalterischen, konstruktiven und wirtschaftlichen Nachteile zu vermeiden, wurden in der Vergangenheit mehrere Versuche unternommen, die Wärmeleitfähigkeit von Beton durch die Zugabe verschiedener Leichtzuschläge soweit zu reduzieren, dass die Herstellung 
einschaliger Außenwände aus Beton möglich ist. Hierfür wurden zunächst konventionelle Leichtbetone verwendet, die unter Zugabe mineralischer oder vulkanischer Leichtzuschläge wie Blähton, Blähschiefer, Blähglas, Perlite oder Naturbims hergestellt werden. Anhand der Unterscheidung zwischen Leichtbetonen mit haufwerksporigem Gefüge, bei denen eine Herabsetzung der Rohdichte und damit der Wärmeleitfähigkeit im Vordergrund steht, und Leichtbetonen mit dichtem Gefüge, die im Hinblick auf die Tragfähigkeit mit Normalbetonen vergleichbar sind, wird die schwierige Verknüpfung der Ziele „niedrige Wärmeleitfähigkeit“ und „hohe Tragfähigkeit“ auch begrifflich deutlich. Entwicklungen aus jüngerer Zeit haben eine Verbesserung des Verhältnisses zwischen Druckfestigkeit und Wärmeleitfähigkeit und damit eine Aufhebung dieser Grenzen zum Ziel. Solche Neuentwicklungen sind z.B. der sog. Infraleichtbeton [2][3], der sog. Architekturleichtbeton [4] oder auch Aerogelbeton [5]-[8]. Bei den Druckfestigkeitsprüfungen von Infraleichtbetonen wurden Druckfestigkeiten erzielt, die eine Einstufung in die Festigkeitsklasse LC8/9 gestatten, wobei die Wärmeleitfähigkeit ca. 0,180,19 W/(mK) beträgt [3]. Die Druckfestigkeit von Architekturleichtbeton liegt unterhalb der Festigkeitsklasse LC8/9, die Wärmeleitfähigkeit wird mit 0,15-0,16 W/(mK) angegeben [4]. Die bislang hergestellten Aerogelbetone weisen entweder sehr niedrige Wärmeleitfähigkeiten $\lambda \leq 0,10 \mathrm{~W} /(\mathrm{mK})$ [5][6] mit korrespondierenden Druckfestigkeiten (Prismenfestigkeit) $f_{\mathrm{lcm}} \leq 3,0 \mathrm{MPa}$ auf, oder erzielen bei Wärmeleitfähigkeiten $\lambda \geq 0,55 \mathrm{~W} /(\mathrm{mK})$ Druckfestigkeiten, die im Bereich der Festigkeitsklassen LC12/13 - LC16/18 liegen [7][8]. Es wird deutlich, dass diese modernen Leichtbetone zwar grundsätzlich die Herstellung tragender, einschaliger Außenwände gestatten, aber bislang aufgrund der niedrigen Druckfestigkeiten oder/und der im Vergleich zu anderen Wandbaustoffen (z.B. Wärmedämmmauerwerk) hohen Wärmeleitfähigkeiten große Wanddicken von $\geq 0,50 \mathrm{~m}$ erfordern. Ziel des vorgestellten Forschungsvorhabens war es daher, einen optimierten Hochleistungsaerogelbeton zu entwickeln, der ein deutlich günstigeres Verhältnis zwischen Druckfestigkeit und Wärmeleitfähigkeit aufweist und somit die Herstellung von tragenden einschaligen Außenwänden mit kleineren Wanddicken ermöglicht. 


\section{Stand der Forschung}

Die grundsätzliche Eignung von Quarzglas-Aerogelgranulat als Zuschlag für Leichtbeton wurde erstmals von Ratke [5] nachgewiesen. Für die Herstellung des Aerogelbetons wurde superhydrophobes Silica-Aerogelgranulat mit einer Partikelgröße von $0,01-4,0 \mathrm{~mm}$, einer Porosität $>90 \%$ und einer Partikelrohdichte von $120-150 \mathrm{~kg} / \mathrm{m}^{3}$ verwendet, das in Anteilen von $50-70 \mathrm{Vol} .-\%$ Mischungen für normalfeste Betone aus CEM II 32,5R, CEM I 42,5R und CEM I 52,5R zugegeben wurde. Anhand von Schliffbildern konnte gezeigt werden, dass die Aerogelpartikel gleichmäßig im Festbeton verteilt und allseitig fest von der Zementmatrix umhüllt sind. Die Ergebnisse der mechanischen und bauphysikalischen Untersuchungen sind in Tabelle 1 zusammengefasst. Hervorzuheben sind die sehr niedrige Wärmeleitfähigkeit, eine hohe Schallabsorption und die hervorragende Eignung als Brandschutzmaterial. Dem stehen eine sehr niedrige Druckfestigkeit und ein sehr geringer Elastizitätsmodul gegenüber. Für den Zusammenhang zwischen Rohdichte und Druckfestigkeit gibt Ratke [5] näherungsweise eine modifizierte Form der Gleichung von Gibson und Ashby [9] an:

$$
f_{l c} \approx 0,2 \sigma_{c r}^{0}\left(\frac{\rho}{\rho_{0}}\right)^{0,75}
$$

Darin ist $\sigma_{c r}^{0}$ die Druckfestigkeit der Zementmatrix, $\rho$ die Dichte des Aerogelbetons und $\rho_{0}$ die Dichte des Zements.

Eine Verbesserung der mechanischen Eigenschaften wurde bei den in [6] beschriebenen Untersuchungen erzielt, indem das Aerogelgranulat in UHPC-Matrizen eingebettet wurde. Dabei wurde ebenfalls hydrophobes Quarzglas-Aerogel-granulat mit Durchmessern 0,01-4,0 mm verwendet, das den Mischungen mit einem Anteil von 65 - 75 Vol.-\% zugegeben wurde, um die Zielrohdichte von ca. $650 \mathrm{~kg} / \mathrm{m}^{3} \mathrm{zu}$ erreichen. Wie Tabelle 1 zeigt, konnte die Druckfestigkeit bei gleichen oder niedrigeren Wärmeleitfähigkeiten durch den Einsatz von UHPC-Mischungen etwa verdoppelt werden. Eine weitere Verdopplung der Druckfestigkeit auf 5,3 MPa konnte erzielt werden, indem die Schwindneigung des 
Aerogelbetons durch eine angepasste Mischungszusammensetzung verringert wurde. Eine Wärmeleitfähigkeit wird für diese Mischung nicht angegeben. Insgesamt bleiben die Festigkeiten unter dem für die praktische Anwendung von Betonen relevanten Festigkeitsbereich. Die weiteren Untersuchungen zeigen, dass Aerogelbeton gegenüber Normalbeton einen etwa $50 \%$ niedrigeren Temperaturausdehnungskoeffizienten, eine erhöhte Schwindneigung und einen hohen Frostwiderstand aufweist. Die Untersuchung des Verbundverhaltens von Betonstahl $\varnothing 8 \mathrm{~mm}$ ergab, dass die maximalen Verbundspannungen gegenüber Normalbeton erheblich herabgesetzt sind und der Verbund ausschließlich über Haftung erfolgt.

Die Idee, für die Herstellung von Aerogelbeton hochfeste Zementmatrizen zu verwenden, wurde ebenfalls in [7] verfolgt. Hierzu wurden Trockenmischungen aus hydrophobem Aerogelgranulat mit einer Partikelgröße von 2 - 4 mm und einer Partikelrohdichte von 100 kg/m³, CEM I 52,5R, Silicastaub und Sand 0,5-2 mm hergestellt und im Anschluss mit destilliertem Wasser und Betonverflüssiger vermischt. Der Aerogelanteil wurde in Zehnprozent-Schritten von $0-60$ Vol.-\% variiert. Für jede Mischung wurde zunächst an Prismen die Biegezugfestigkeit bestimmt, aus den Bruchstücken wurden dann Probekörper für die Ermittlung der Druckfestigkeit und der Wärmeleitfähigkeit gewonnen. Die Ergebnisse der Untersuchungen sind in Tabelle 1 angegeben. Für die Mischung mit dem höchsten Aerogelanteil von 60 Vol.-\% und einer korrespondierenden Rohdichte von $1000 \mathrm{~kg} / \mathrm{m}^{3}$ wurde eine Wärmeleitfähigkeit von $0,26 \mathrm{~W} /(\mathrm{mK})$, eine Druckfestigkeit von 8,3 $\mathrm{MPa}$ und eine Biegezugfestigkeit von 1,2 MPa ermittelt. Aus den weiteren Ergebnissen wurden folgende Beziehungen für den Zusammenhang zwischen Rohdichte und Druckfestigkeit sowie zwischen Rohdichte und Wärmeleitfähigkeit abgeleitet:

$$
\begin{aligned}
& f_{l c}=0,57 e^{0,0023 \rho} \\
& \lambda=0,038 e^{0,0019 \rho}
\end{aligned}
$$

Darin ist $f_{\mathrm{lc}}$ die Prismendruckfestigkeit in $\mathrm{MPa}, \lambda$ die Wärmeleitfähigkeit in $\mathrm{W} /(\mathrm{mK})$ und $\rho$ die Rohdichte des Aerogelbetons in $\mathrm{kg} / \mathrm{m}^{3}$. 
Bei den in [8] durchgeführten Untersuchungen an UHPC mit Aerogelgranulat als Zuschlag entsprachen die Vorgehensweise und der Untersuchungsumfang weitgehend den zuvor beschriebenen Versuchen aus [7]. Es wurde jedoch Aerogelgranulat mit einer Partikelgröße von 0,01 - $4 \mathrm{~mm}$ verwendet, das den Mischungen erst am Ende des Mischvorgangs in Anteilen von 0, 20, 40, 50, 60, 70 und 80 Vol.-\% von Hand beigemischt wurde. Der Wasser-Zement-Wert konnte von ca. 0,4 auf ca. 0,2 reduziert werden, indem der Anteil des Betonverflüssigers erhöht wurde. Die resultierenden Druckfestigkeiten, Biegezugfestigkeiten und Wärmeleitfähigkeiten für die UHPC-Mischungen können ebenfalls Tabelle 1 entnommen werden. Es wurde festgestellt, dass für einen Aerogelbeton, der eine mit Normalbetonen vergleichbare Druckfestigkeit von $20 \mathrm{MPa}$ aufweist, ein Aerogelanteil von $50 \mathrm{Vol.- \%}$ verwendet werden sollte. Für diese Mischung wurden eine Rohdichte von ca. $1.350 \mathrm{~kg} / \mathrm{m}^{3}$, eine Biegezugfestigkeit von $5 \mathrm{MPa}$ und eine Wärmeleitfähigkeit von $0,55 \mathrm{~W} /(\mathrm{mK})$ ermittelt. Für Aerogelgehalte von 60 bzw. 70 Vol.-\% wurde ein Abfall der Druckfestigkeit auf ca. $10 \mathrm{MPa}$ bzw. ca. $5 \mathrm{MPa}$ beobachtet, während die Wärmeleitfähigkeiten sich nur auf ca. 0,5 bzw. 0,45 W/(mK) verbesserten. Für Mischungen mit $80 \%$ Aerogelanteil konnte keine messbare Druckfestigkeit bestimmt werden. Daher wird in [8] empfohlen, für die Herstellung tragfähiger Aerogelbetone Mischungen mit einem Aerogelanteil von $50-60$ Vol.-\% zu wählen. Für Probekörper aus parallel hergestellten Zement-Silica-Mischungen, die ohne die für UHPC typischen Feinbestandteile hergestellt wurden, wurden bei gleichen Aerogelanteilen geringere Druckfestigkeiten und Wärmeleitfähigkeiten beobachtet.

\section{Mischungszusammensetzung und Mischvorgang}

\subsection{Voruntersuchungen}

Um grundsätzliche Erkenntnisse zur Mischungszusammensetzung und zur Mischreihenfolge zu gewinnen, wurden zunächst unter Verwendung von Referenzrezepturen für normal-, hoch- und ultrahochfeste Betone über 40 verschiedene Aerogelbetonmischungen hergestellt. An den daraus gefertigten Prismen mit 40 mm x 40 mm x 160 mm Kantenlänge wurde jeweils die Biegezug- und 
Druckfestigkeit bestimmt. Bei diesen Voruntersuchungen wurden folgende grundsätzliche Zusammenhänge beobachtet:

- Die Rohdichte und die Druckfestigkeit korrelieren mit dem Aerogelanteil.

- Mit HPC- und insbesondere UHPC-Mischungen werden größere Druckfestigkeiten erzielt als mit Normalbetonmischungen.

- Mischungen, bei denen die Gesteinskörnung gegen Aerogelgranulat ausgetauscht wurde, weisen eine geringere Rohdichte, eine fließfähigere Konsistenz und eine höhere Druckfestigkeit auf als die Mischungen, denen Aerogel zusätzlich zur Gesteinskörnung zugegeben wurde.

- Im Gegensatz zu den Beobachtungen aus [8] wurden für die Mischungen, die ohne Quarzsand, aber mit höherem Zementgehalt hergestellt wurden, höhere Druckfestigkeiten ermittelt als für Mischungen mit Quarzsand und geringerem Zementgehalt. Ursächlich hierfür sind vermutlich die höheren w/z-Werte in [8], die aus einer Reduzierung des Fließmittelanteils resultierten.

Auf Grundlage dieser Voruntersuchungen wurden optimierte Mischungen hergestellt, aus denen Würfelprobekörper mit 150 mm Kantenlänge gefertigt wurden. Die erforderlichen Aerogelbetonmengen wurden unter Verwendung eines $150 \mathrm{~L}$ - Intensivmischers bzw. eines $60 \mathrm{~L}$ - Gleichlaufmischers gemischt. Hierbei wurden Informationen über die Konsistenz und Verarbeitbarkeit der verschiedenen Mischungen bei der Produktion größerer Mengen gewonnen. Der Aerogelanteil wurde hierbei zwischen 40 und 60 Vol.-\% variiert, zudem wurden der Einfluss von zusätzlichen Leichtzuschlägen (Leichtsand und Blähton) und eine Variation der Silicazugabe (als Staub oder Suspension) untersucht. Je Mischung wurden 18 Würfelprobekörper hergestellt, um den Einfluss der Lagerungsbedingungen zu untersuchen und die Druckfestigkeit nach sieben und 28 Tagen zu bestimmen. Jeweils sechs Würfel wurden gemäß [10] einer Trockenlagerung und einer gemischten Lagerung unterzogen. Ein Großteil der Würfel musste hierbei beschwert werden, da ansonsten aufgrund der Rohdichten $<1.000 \mathrm{~kg} / \mathrm{m}^{3}$ ein Aufschwimmen der 
Würfel festzustellen war. Weitere sechs Würfel wurden im Alter von 24 bis 48 Stunden bei $90{ }^{\circ} \mathrm{C}$ im Trockenschrank gelagert. Die Ergebnisse der Druckfestigkeitsprüfungen zeigten:

- Die größten Druckfestigkeiten konnten im Regelfall für die Würfel, die der gemischten Lagerung unterzogen wurden, beobachtet werden.

- Eine Wärmebehandlung hatte keinen eindeutigen Einfluss auf die Druckfestigkeit.

- Bei einem Vergleich der Druckfestigkeiten im Alter von sieben und 28 Tagen war kein eindeutiger Trend im Hinblick auf die Festigkeitsentwicklung zu beobachten.

- Die Verwendung von Silicastaub führte im Vergleich zu Silicasuspension - unter Berücksichtigung des Wasseranteils in der Suspension - zu größeren erforderlichen w/z-Werten. Hieraus resultierten geringere Druckfestigkeiten.

- Eine deutliche Reduzierung des w/z-Wertes und eine Steigerung der Druckfestigkeit konnten festgestellt werden, wenn das Zugabewasser auf $\leq 5^{\circ} \mathrm{C}$ abgekühlt wurde. Die so hergestellten Mischungen waren fließfähig, auch bei hohen Aerogelanteilen ( $>65 \%)$ selbstverdichtend und dadurch sehr gut verarbeitbar.

Die Probekörper wurden jeweils im Alter von 24 Stunden ausgeschalt und wiesen zu diesem Zeitpunkt bereits erhöhte Schwindverformungen auf. Da ein Zusammenhang zwischen Schwindverformung und Druckfestigkeit vermutet wurde, wurde in einer weiteren Testreihe der Einfluss einer Glasfaserzugabe untersucht. Die mit Glasfaseranteilen von 0,5-2,0 Vol.-\% hergestellten Mischungen hatten eine sehr steife Konsistenz und waren schlecht $\mathrm{zu}$ verarbeiten. Da zwar ein positiver Effekt auf die Schwindverformung, aber kein signifikanter Einfluss auf die Druckfestigkeit feststellbar war, wurde dieser Ansatz nicht weiter verfolgt. 


\subsection{Festlegung einer Referenzmischung}

Basierend auf den Ergebnissen aus über 70 verschiedenen Aerogelbetonmischungen wurde eine Referenzmischung konzipiert, die für die weiteren Untersuchungen herangezogen wurde. Die zugehörige Mischungszusammensetzung ist in Tabelle 2 angegeben.

Als Aerogelgranulat kam hydrophobes Quarzglas-Aerogelgranulat zum Einsatz, das eine Partikelgröße von $0,01-4,0 \mathrm{~mm}$, eine Porosität $>90 \%$ und eine Partikelrohdichte von $100 \mathrm{~kg} / \mathrm{m}^{3}$ aufweist. Die Wärmeleitfähigkeit dieses Aerogels beträgt bei Raumtemperatur $\left(20^{\circ} \mathrm{C}\right) \leq 0,02 \mathrm{~W} /(\mathrm{mK})$.

Für die Untersuchungen der mechanischen und bauphysikalischen Eigenschaften wurden auf Grundlage dieser Referenzmischung Probekörper mit 50, 60 und 70 Vol.-\% Aerogelanteil hergestellt. Hierbei wurde jeweils ein definiertes Mischregime verwendet. Aus den Probekörpern wurden Scheiben gesägt, mit denen Schliffbilder angefertigt wurden. Wie auf der Mikroskopieaufnahme in Bild 1 zu erkennen ist, wurden mit der beschriebenen Mischungszusammensetzung und dem Mischregime Mischungen mit einer homogenen Verteilung des Aerogelgranulats und einer vollständigen Einbettung der Aerogelpartikel in die UHPC-Matrix erzeugt.

\section{Untersuchungen der mechanischen Eigenschaften}

\subsection{Rohdichte}

Die in [5] - [8] beschriebene Korrelation von Aerogelanteil und Rohdichte wurde im Rahmen dieser Arbeit bestätigt. Wie aus Bild 2 zu erkennen ist, sind die Trockenrohdichten der am IfM hergestellten Probekörper bei gleichen Aerogelanteilen jedoch kleiner als in [7] und [8]. Dies ist darauf zurückzuführen, dass die Mischungen ohne (Fein-)Sande hergestellt wurden und somit geringere Feststoffanteile aufweisen als die in [7] und [8] beschriebenen Mischungen.

Der Zusammenhang zwischen Aerogelanteil und Trockenrohdichte kann in guter Näherung durch einen linearen Ansatz beschrieben werden (siehe auch Bild 2): 


$$
\rho=2820-32 \varphi
$$

Darin in $\rho$ die Trockenrohdichte in $\mathrm{kg} / \mathrm{m}^{3}$ und $\varphi$ der Aerogelanteil in Vol.-\%.

\subsection{Druckfestigkeit}

Die Druckfestigkeitsprüfungen wurden gemäß [11] an einer 3MN-Zweisäulen-Druckprüfmaschine durchgeführt. Die Würfelprobekörper mit einer Kantenlänge von $150 \mathrm{~mm}$ wurden nach [12] hergestellt und gemäß [13] gelagert. Aufgrund der zu erwartenden, im Vergleich zu Normalbeton herabgesetzten Druckfestigkeiten wurde die Belastungsgeschwindigkeit nach [11] auf den unteren Grenzwert von 0,4 N/(mm²s) festgelegt. Dies entspricht bei einer Prüffläche von $150 \mathrm{~mm} \mathrm{x} 150 \mathrm{~mm}$ einer Belastungsgeschwindigkeit von $9 \mathrm{kN} / \mathrm{s}$, die durch Einsatz einer elektronischen Steuerung gewährleistet wurde. Bild 3 zeigt, dass das Bruchbild der Aerogelbetonwürfel mit dem von Normalbeton vergleichbar und nach [11] als ,zufriedenstellendes Bruchbild“ einzustufen ist.

Insgesamt wurden Druckfestigkeitsprüfungen an Probekörpern aus 52 Mischungen durchgeführt. In Bild 4 ist der Zusammenhang zwischen der Trockenrohdichte und den Mittelwerten der Betondruckfestigkeit aufgetragen. Analog zu den Untersuchungen in [7] kann dieser Zusammenhang durch eine Exponentialfunktion beschrieben werden:

$$
f_{\text {lcm,cube } 150}=0,37 e^{0,0034 \rho}
$$

Darin ist $f_{\text {lcm,cube150 }}$ in $\mathrm{MPa}$ und $\rho$ in $\mathrm{kg} / \mathrm{m}^{3}$ einzusetzen. Da die in [5] - [8] angegebenen Druckfestigkeitswerte an Prismen mit $40 \mathrm{~mm}$ Kantenlänge und die am IfM erzielten Druckfestigkeitswerte an Würfeln mit $150 \mathrm{~mm}$ Kantenlänge ermittelt wurden, sind die Werte nicht direkt vergleichbar. Um zumindest näherungsweise einen Vergleich zu ermöglichen, wurden die Druckfestigkeiten aus [5] - [8] in Bild 4 mit dem Faktor 0,9 auf die Druckfestigkeit von Würfeln mit $150 \mathrm{~mm}$ Kantenlänge umgerechnet. Dieser Umrechnungsfaktor wurde durch eigene Vergleiche der 
Prismen- und Würfeldruckfestigkeiten von Aerogelbetonprobekörpern bestimmt und bestätigt die in [14] und [15] angegebenen Werte für Leichtbetone bzw. hochfeste Mörtel. Auf dieser Grundlage zeigt Bild 4, dass die Druckfestigkeiten der am IfM entwickelten Hochleistungsaerogelbetone für Rohdichten $<600 \mathrm{~kg} / \mathrm{m}^{3}$ vergleichbar mit denen aus [5] - [8] sind, für Rohdichten $\geq 600 \mathrm{~kg} / \mathrm{m}^{3} \mathrm{jedoch}$ erheblich höher.

\subsection{Biegezugfestigkeit}

Die Biegezugfestigkeit nach [16] wurde für drei Aerogelbetonmischungen mit ca. 50, 60 und 70 Vol.-\% Aerogelanteil bestimmt (M48, M50, M52). Hierzu wurden Vier-Punkt-Biegeversuche an Probekörpern mit den Abmessungen 700 mm x 150 mm x 150 mm (LxBxH) durchgeführt. Zum Vergleich wurde die Biegezugfestigkeit für Probekörper aus einer Leichtbetonmischung der Festigkeitsklasse LC8/9 ( $\rho=1360 \mathrm{~kg} / \mathrm{m}^{3}$ ) ermittelt, die unter Verwendung von Leichtsand und Blähton hergestellt wurde (M47). Die Ergebnisse sind in Tabelle 3 angegeben. Die vorhandene Datenbasis erlaubt keine abschließende Einordnung der Biegezugfestigkeit. Bezogen auf die Rohdichte ergeben sich für die untersuchten Aerogelbeton-Biegebalken Werte, die mit denen von Leichtbeton vergleichbar sind. Dies gilt auch für einen Vergleich mit den in [6] - [8] angegebenen Biegezugfestigkeiten. Dabei ist, wie bereits erwähnt, zu beachten, dass die Biegezugversuche dort an Prismen mit 40 mm x 40 mm x 160 mm Kantenlänge durchgeführt wurden. Werden die am IfM ermittelten Biegezugfestigkeiten nach einem in [14] für Leichtbetone angegebenen Vorschlag auf die zentrische Zugfestigkeit umgerechnet, so ergibt sich für die untersuchten Probekörper $f_{\text {lctm }}=0,77 \cdot f_{\text {lct,fl }}$. Tab. 3 zeigt, dass die so ermittelte zentrische Zugfestigkeit, ähnlich wie bei Normalbetonen, näherungsweise 5-10\% der Würfeldruckfestigkeit beträgt. 


\subsection{Elastizitätsmodul}

Für die Mischungen M48, M50 und M52 wurde außerdem der Elastizitätsmodul an zylindrischen Probekörpern mit einem Durchmesser von 150 mm und einer Höhe von 300 mm nach [17] bestimmt. Aufgrund der im Verhältnis zu normalfesten Betonen herabgesetzten Druckfestigkeit des Aerogelbetons wurden die Belastungszyklen zur Bestimmung des E-Moduls nach [18] gewählt. Die ermittelten EModuli (Tabelle 3) sind z.T. erheblich größer als die in [5] und [6] angegebenen, was auf die höheren Betondruckfestigkeiten der am IfM entwickelten Hochleistungs-Aerogelbetone zurückgeführt werden kann. Der mittlere E-Modul $\left(E_{\mathrm{lcm}}\right)$ von Hochleistungsaerogelbeton kann für Druckfestigkeiten $\geq 5 \mathrm{MPa}$ näherungsweise in Abhängigkeit von der Druckfestigkeit und der Trockenrohdichte nach [19] bestimmt werden:

$$
E_{l c m}=\left(\frac{\rho}{2200}\right)^{2} \cdot 22000 \cdot\left(\frac{f_{l c m}}{10}\right)^{0,3}
$$

mit $\rho$ in $\mathrm{kg} / \mathrm{m}^{3}$ und $f_{\text {lcm }}$ sowie $E_{\mathrm{lcm}}$ in $\mathrm{MPa}$.

\section{Untersuchungen der bauphysikalischen Eigenschaften}

\section{$5.1 \quad$ Wärmeleitfähigkeit}

Zur Ermittlung der Wärmeleitfähigkeit wurden Würfel mit einer Kantenlänge von $150 \mathrm{~mm}$ hergestellt und im Alter von 14 Tagen in Scheiben mit einer Dicke von 30 mm gesägt. Nach dem Sägen wurden die Probekörper mindestens 24 Stunden bei $100{ }^{\circ} \mathrm{C}$ im Trockenschrank gelagert. Die Bestimmung der Wärmeleitfähigkeit erfolgte nach [20] mit einem Wärmestrommessplatten-Gerät [21]. Zwar wurde auch hierbei eine Korrelation zwischen Druckfestigkeit und Wärmeleitfähigkeit beobachtet. Wie aus Bild 5 erkennbar ist, sind die ermittelten Wärmeleitfähigkeiten der am IfM entwickelten Hochleistungsaerogelbetone im Verhältnis zur Druckfestigkeit jedoch deutlich kleiner als die für die 
Aerogelbetone in [6] und [8] angegebenen Werte. Analog zu Abs. 4.2 wurden die Druckfestigkeiten aus [6] und [8] mit dem Faktor 0,9 umgerechnet. Für den hier untersuchten Festigkeitsbereich $2 \mathrm{MPa} \leq f_{\text {lcm,cube150 }} \leq 25 \mathrm{MPa}$ kann der Zusammenhang zwischen Druckfestigkeit und Wärmeleitfähigkeit näherungsweise linear beschrieben werden:

$$
\lambda=0,006 \cdot f_{\text {lcm, cube } 150}+0,11
$$

mit $\lambda$ in $\mathrm{W} /(\mathrm{mK})$ und $f_{\mathrm{lcm}, \text { cube150 }}$ in $\mathrm{MPa}$.

\subsection{Schallabsorption}

Zur Bewertung des Schalldämmvermögens wurde für drei Aerogelbetonmischungen mit ca. 50, 60 und ca. 70 Vol.-\% Aerogelanteil und zum Vergleich für drei Proben aus Leicht- und Normalbeton der materialspezifische Absorptionskoeffizient $\mu$ bestimmt, der einen dickenunabhängigen Vergleich des Schalldämmvermögens verschiedener Materialien ermöglicht.

Die Messungen wurden an einem eigens vom DLR entwickelten Versuchsstand mit dem Transmissionsverfahren durchgeführt. Für die Herstellung der Probekörper wurden Aerogelbetonwürfel in Scheiben mit einer Dicke von 20 mm gesägt. Die Ergebnisse der Untersuchungen sind in Tabelle 4 dargestellt.

Bei einem Vergleich des Leichtbetons (M41), des Normalbetons und der Aerogelbetone M42 und M51 ist zu erkennen, dass die Aerogelbetone trotz der auf 20 - $40 \%$ reduzierten Rohdichten mit Leicht- oder Normalbetonen vergleichbare Schallabsorptionskoeffizienten erzielen $(\mu=0,224-0,2411 / \mathrm{cm})$. Der Aerogelbeton M43 weist im Vergleich zum Normalbeton bei einer ca. 40 \% geringeren Rohdichte mit $\mu=0,3091 / \mathrm{cm}$ ein um ca. $38 \%$ besseres Schallabsorptionsvermögen auf. Die entwickelten Hochleistungsaerogelbetone zeigen im Versuch somit trotz der erheblich kleineren Rohdichten ein hervorragendes Schallschutzvermögen. 


\section{Weiterführende Untersuchungen}

\subsection{Bauteile aus gradiertem Aerogelbeton}

Im Hinblick auf eine industrielle Herstellung von Außenwänden wurde die Herstellung von Fertigteilelementen aus gradiertem Aerogelbeton untersucht. Diese Fertigteile bestehen aus einer Tragschale mit hoher Druckfestigkeit und verhältnismäßig niedriger Wärmeleitfähigkeit und einer nichttragenden Außenschale mit sehr niedriger Wärmeleitfähigkeit. Zielsetzung ist, durch diesen gradierten bzw. gestuften Wandaufbau frei formbare Fertigteile herzustellen, die die Tragfähigkeit von Wärmedämmmauerwerk bei gleichen oder reduzierten U-Werten übertreffen und somit die Herstellung von tragenden Außenwänden ermöglichen, die den bauphysikalischen Anforderungen der ENEV 2014 [22] genügen.

Die grundsätzliche Eignung von gradierten Aerogelbetonbauelementen wurde anhand von experimentellen Untersuchungen überprüft. Auf Basis der in Tab. 2 angegebenenMischung wurden fließfähige und selbstverdichtende Mischungen für die Trag- und die Dämmschale hergestellt. Für die wärmedämmende Schicht wurde eine Mischung mit einem Aerogelanteil von 68,3 Vol.-\% und einem w/z-Wert von $0,48\left(f_{\text {lcm,cube }}=2,1 \mathrm{MPa}\right)$, für die Tragschale eine Mischung mit einem Aerogelanteil von 47,0 Vol.-\% und einem w/z-Wert von 0,24 ( $\left.f_{\text {lcm,cube } 150}=19,5 \mathrm{MPa}\right)$ hergestellt. Aus diesen Mischungen wurden im „frisch-auf-fest-Verfahren“ gradierte Probekörper angefertigt (Bild 6), an denen der BetonBeton-Verbund untersucht wurde.

Die Verbundfuge zwischen nichttragender Außenschale und tragender Innenschale wird durch das Eigengewicht der Außenschale (Belastung parallel zur Verbundfuge) und durch Windsog (Belastung senkrecht zur Verbundfuge) beansprucht. Daher wurde der Beton-Beton-Verbund mittels Scherversuch und Haftzugversuch getestet. Die ermittelten Verbund- und Haftzugfestigkeiten liegen erheblich über den baupraktisch zu erwartenden Beanspruchungen. Unter Annahme einer $30 \mathrm{~cm}$ dicken Dämmschale mit einer Rohdichte von $1000 \mathrm{~kg} / \mathrm{m}^{3}$ ergibt sich parallel zur Verbundfuge ein Ausnutzungsgrad von ca. 2,5\%. Für Bauwerke mit einer maximalen Windsogbeanspruchung von $5 \mathrm{kN} / \mathrm{m}^{2}$ beträgt der 
Ausnutzungsgrad senkrecht zur Verbundfuge ca. 8,3\%. Unter Berücksichtigung dieser Ergebnisse ist eine ausreichende Tragfähigkeit auch bei Einführung von Fraktil- und Teilsicherheitsbeiwerten nachweisbar.

Der Nachweis der grundsätzlichen Eignung von Hochleistungsaerogelbeton für die Herstellung gradierter Bauelemente eröffnet gänzlich neue Möglichkeiten für die Fertigung von wärmedämmenden Außenbauteilen aus Beton. So ist es beispielsweise denkbar, ein Fertigteilelement aus einer $120 \mathrm{~mm}$ dicken Tragschale $(\lambda=0,26 \mathrm{~W} /(\mathrm{mK}))$ und einer $80 \mathrm{~mm}$ dicken Dämmschale $(\lambda=0,09 \mathrm{~W} /(\mathrm{mK}))$ herzustellen. Unter Berücksichtigung eines auf der Innen- und Außenseite aufgebrachten Aerogeldämmputzes mit 30 mm Dicke [23] ergibt sich für diesen Wandaufbau mit einer Fertig-Gesamtdicke von $26 \mathrm{~cm}$ ein U-Wert von $0,27 \mathrm{~W} /\left(\mathrm{m}^{2} \mathrm{~K}\right)$. Somit könnten ENEV-konforme Außenwände von Wohn- oder Nichtwohngebäuden hergestellt werden, deren Dicke die von Leichtbetonwänden, Wärmedämmmauerwerk oder Betonwänden mit einem Wärmedämmverbundsystem bei erheblich größerer Tragfähigkeit unterschreitet.

\subsection{Aerogelbeton mit GFK-Bewehrung}

Analog zu Normalbeton ist bei dem entwickelten Hochleistungsaerogelbeton unter Biegebeanspruchung zur Aufnahme von Zugkräften eine Bewehrung erforderlich. Dazu wurden bereits in [6] Untersuchungen mit konventioneller Betonstahlbewehrung durchgeführt. Hierbei wurden nur geringe Verbundspannungen ermittelt, die offensichtlich auf die niedrigen Betondruckfestigkeiten und den damit einhergehenden, nur schwach ausgeprägten Scherverbund zurückzuführen sind. Bei den experimentellen Untersuchungen an Hochleistungsaerogelbeton durch das IfM wurde aus verschiedenen Gründen eine GFK-Bewehrung [24] verwendet. Zum Einen erscheint diese Wahl wegen einer möglichen negativen Beeinflussung der hervorragenden Wärmedämmeigenschaften des Aerogelbetons durch Betonstahl sinnvoll, denn GFK-Bewehrung weist mit $\lambda=0,5 \mathrm{~W} /(\mathrm{mK})$ in radialer bzw. $\lambda=0,7 \mathrm{~W} /(\mathrm{mK})$ in axialer Richtung eine erheblich niedrigere Wärmeleitfähigkeit auf als Betonstahl 
$(\lambda \approx 60 \mathrm{~W} /(\mathrm{mK}))$. Zum anderen wurde bei den Untersuchungen in [6] festgestellt, dass der Temperaturausdehnungskoeffizient von Aerogelbeton nur $\alpha_{\mathrm{T}}=0,5 \cdot 10^{-5} 1 / \mathrm{K}$ und damit nur ca. $50 \%$ der Werte von Normalbeton und Betonstahl beträgt. Die GFK-Bewehrung $\left(\alpha_{\mathrm{T}}=0,6 \cdot 10^{-5} 1 / \mathrm{K}\right)$ wurde daher auch zur Vermeidung innerer Spannungen, die aus unterschiedlichen Temperaturausdehnungskoeffizienten resultieren können, eingesetzt.

Zur Ermittlung der Verbundspannungen wurden an fünf Serien mit je sechs Probekörpern Pull-OutTests in Anlehnung an [25] durchgeführt. Die Serien unterschieden sich hinsichtlich der Mischungszusammensetzungen. Eine Serie wurde ohne die Zugabe von Glasfasern, zwei Serien mit Glasfasern und zwei Serien mit Leichtzuschlägen und Glasfasern hergestellt. Die GFK-Stäbe (Ø 12 mm und $\varnothing 16 \mathrm{~mm}$ ) wurden in $18 \mathrm{~cm}$ x $18 \mathrm{~cm}$ x $30 \mathrm{~cm}$ große Aerogelbetonquader einbetoniert, wobei die Verbundlänge 14,4 cm betrug, die verbundfreie Vor- und Nachlänge variierte in Abhängigkeit vom Stabdurchmesser. Die Versuche wurden an einer Zugprüfmaschine mit einer maximalen Prüfkraft von $600 \mathrm{kN}$ weggesteuert mit einer Belastungsgeschwindigkeit von $0,3 \mathrm{~mm} / \mathrm{min}$ durchgeführt.

Alle Probekörper wiesen ein für Beton typisches Verbundverhalten nach [26] auf, d.h., nach dem Versagen des Haftverbundes wurde der Scherverbund aktiviert, der bei einem Schlupf von ca. 2 - 3 mm versagte. Die ermittelten Verbundspannungen sind in Abhängigkeit von der jeweiligen Druckfestigkeit der Hochleistungsaerogelbetone in Bild 7 aufgetragen. Zum Vergleich sind dort auch die Verbundspannungen für Aerogelbeton mit Betonstahlbewehrung aus [27] sowie für Infraleichtbeton [2] angegeben.

Es wird deutlich, dass sowohl für den Aerogelbeton aus [27] als auch für den Hochleistungsaerogelbeton des IfM der Zusammenhang zwischen Druckfestigkeit und dem Mittelwert der maximalen Verbundspannungen analog zu Normal- oder Leichtbeton durch eine Potenzialfunktion ausgedrückt werden kann (Bild 7). In Anlehnung an die in [28] angegebene Gleichung für Leichtbeton kann dieser Zusammenhang bei gutem Verbund näherungsweise durch folgende Gleichung ausgedrückt werden: 


$$
\tau_{\max , m}=0,38 \cdot f_{l c m, c u b e 150}{ }^{0,82}
$$

In Bild 7 ist ebenfalls das Verhältnis zwischen mittlerer Druckfestigkeit und maximaler Verbundspannung für Leichtbeton nach [28] dargestellt. Demzufolge sind die Verbundspannungen bei Aerogelbetonen etwas kleiner als bei Leichtbetonen. Sie sind jedoch größer als beispielsweise bei Infraleichtbeton [2] und insgesamt auf einem Niveau, das die Herstellung bewehrter Aerogelbetone erlaubt. Derzeit werden am IfM diesbezüglich weitere Untersuchungen durchgeführt.

\section{Zusammenfassung und Ausblick}

Durch umfangreiche Untersuchungen an verschiedenen Aerogelbetonen wurde eine Mischungszusammensetzung mit zugehörigem Mischregime gefunden, die die Herstellung von Hochleistungsaerogelbetonen mit einem deutlich günstigeren Zusammenhang zwischen Rohdichte und Druckfestigkeit bzw. Wärmeleitfähigkeit und Druckfestigkeit als bei den bisher entwickelten Aerogelbetonen [5]-[8] ermöglicht. Durch die signifikant erhöhten Druckfestigkeiten wurden erstmalig die Voraussetzungen für den Praxiseinsatz des Werkstoffes Aerogelbeton geschaffen. Dabei ist es gelungen, auch größere Mengen des Werkstoffs zielsicher mit fließfähiger Konsistenz herzustellen, so dass der Aerogelbeton sich gut verarbeiten lässt und in der Regel selbstverdichtend ist.

Die niedrigen Wärmeleitfähigkeiten des Materials gestatten die Herstellung ENEV-konformer, einschaliger Betonaußenwände ohne weitere Wärmedämmung. Hierfür haben sich UHPC-Mischungen mit einem Aerogelanteil von ca. 60 Vol.-\% und einer korrespondierenden Trockenrohdichte von $650 \mathrm{~kg} / \mathrm{m}^{3}<\rho \leq 1090 \mathrm{~kg} / \mathrm{m}^{3}$ als geeignet erwiesen. Die in diese Rubrik fallenden Mischungen (in Bild 4 und 5 blau angelegte Bereiche) weisen mittlere Druckfestigkeiten im Bereich $5,8 \mathrm{MPa} \leq f_{\text {lcm,cube150 }} \leq 14,4 \mathrm{MPa} \quad$ und $\quad$ Wärmeleitfähigkeiten im $\quad$ Bereich $0,17 \mathrm{~W} /(\mathrm{mK}) \leq \lambda \leq 0,19 \mathrm{~W} /(\mathrm{mK})$ auf. Unter Verwendung eines beidseitigen, $30 \mathrm{~mm}$ dicken 
Aerogeldämmputzes lassen sich hiermit ENEV-konforme Außenwände mit einer Dicke (Rohbaumaß) von $36,5 \mathrm{~cm}$ herstellen.

Eine Reduzierung der Wanddicken ist möglich, indem Außenwände aus gradierten Aerogelbetonwandelementen hergestellt werden. Die grundsätzliche Ausführbarkeit solcher Bauelemente, die vorzugweise als Fertigteile herzustellen sind, wurde im Rahmen der Untersuchungen nachgewiesen. Aerogelbetonmischungen für die Tragschichten (in Bild 4 und 5 orange angelegte Bereiche) weisen typischerweise Aerogelanteile von ca. 50 Vol.-\% und damit korrespondierende Trockenrohdichten von $1090 \mathrm{~kg} / \mathrm{m}^{3}<\rho \leq 1340 \mathrm{~kg} / \mathrm{m}^{3}$ auf. Die Druckfestigkeiten liegen im Bereich $19,5 \mathrm{MPa} \leq f_{\text {lcm,cube150 }} \leq 25,5 \mathrm{MPa}$ und die Wärmeleitfähigkeiten bei $0,26 \mathrm{~W} /(\mathrm{mK})$, d.h., bei einer mit Normalbeton vergleichbaren Druckfestigkeit beträgt die Wärmeleitfähigkeit nur ca. $15 \%$ der Wärmeleitfähigkeit von Normalbeton. Für die außenliegende Dämmschicht gradierter Aerogelbetonwände erscheinen Aerogelanteile von ca. 70 Vol.-\% und damit verbunden Trockenrohdichten von ca. $540 \mathrm{~kg} / \mathrm{m}^{3}$ sinnvoll. Solche Mischungen (in Bild 4 und 5 grün angelegt) weisen eine geringe Druckfestigkeit von ca. $2 \mathrm{MPa}$ auf, zeichnen sich aber durch eine Wärmeleitfähigkeit von nur 0,09 W/(mK) aus. Unter Annahme einer $120 \mathrm{~mm}$ dicken Tragschicht und einer $80 \mathrm{~mm}$ dicken Dämmschicht sowie eines beidseitigen, $30 \mathrm{~mm}$ dicken Aerogeldämmputzes ließen sich hiermit ENEV-konforme Außenwände mit einer Dicke von $20 \mathrm{~cm}$ (Rohbaumaß) bzw. $26 \mathrm{~cm}$ (Fertigmaß) herstellen. Die Messungen der Schallabsorption zeigen, dass im Vergleich zu Normalbeton sowohl bei einschaliger als auch bei gradierter Bauweise ein vergleichbares oder besseres Schallschutzniveau erreicht wird.

Da Aerogelbeton verhältnismäßig kleine, mit Leichtbeton vergleichbare Biegezugfestigkeiten aufweist, ist für die Herstellung biegebeanspruchter Aerogelbetonbauteile wie auch bei Normal- oder Leichtbeton eine Bewehrung erforderlich. Aufgrund der bei Auszugversuchen an Aerogelbetonprobekörpern mit Bewehrung aus glasfaserverstärktem Kunststoff ermittelten Verbundspannungen erscheint die Herstellung bewehrter Aerogelbetonbauteile prinzipiell möglich. Damit ist beispielsweise die 
Herstellung von obersten Geschossdecken oder von Tür- und Fensterstürzen in Außenwänden aus Aerogelbeton denkbar. Die erforderlichen weiteren Untersuchungen werden derzeit am IfM vorbereitet.

\section{Danksagung}

Die Autoren bedanken sich für die Förderung des Projekts durch das Land Nordrhein-Westfalen und die Europäische Union (NRW-EFRE Projekt-Nr. 64.65.69-PRO-0057 A).

Literatur

[1] Schnellenbach-Held, M.; Welsch, T.; Fickler, S.; Rhau, M.; Wallner, M.; Ratke, L.; Milow, B.: Aerogelbasierte Baustoffe als dämmendes und tragfähiges Konstruktionsmaterial für Büro- und Laborgebäude. Schlussbericht NRW-EFRE Projekt-Nr. 64.65.69-PRO-0057 A, Essen: 2015.

[2] Schlaich, M.; El Zareef, M.: Infraleichtbeton. Beton- und Stahlbetonbau 103 (2008), H. 3, S. $175-$ 182.

[3] Schlaich, M.; Hückler, A.: Infraleichtbeton 2.0. Beton- und Stahlbetonbau 107 (2012), H. 11, S. 757-766.

[4] Breit, W.; Schulze, J.; Heese, C.; Bund, B.: Hochwärmedämmende, monolithische Sichtbetonaußenteile aus Architekturleichtbeton. Stuttgart: Fraunhofer IRB 2014.

[5] Ratke, L.: Herstellung und Eigenschaften eines neuen Leichtbetons: Aerogelbeton. Beton- und Stahlbetonbau 103 (2008), H. 4, S. 236-243.

[6] Hub, A.; Zimmermann, G.; Knippers, J.: Leichtbeton mit Aerogelen als Konstruktionswerkstoff. Beton- und Stahlbetonbau 108 (2013), H. 9, S. 654-661. 
[7] Gao, T.; Jelle, B. P.; Gustavsen, A.; Jacobsen, S.: Aerogel-incorporated Concrete: An Experimental Study. Construction and Building Materials 52 (2014), S. 130-136.

[8] Ng, S.; Jelle, B. P.; Sandberg, L. I. C.; Gao, T.; Wallevik, O. H.: Experimental Investigations of Aerogel-incorporated Ultra-high Performance Concrete. Construction and Building Materials 77 (2015), S. 307-316.

[9] Gibson, L. J.; Ashby M. F.: Cellular Solids. Cambridge: Cambridge University Press, 2nd. Edition, 1997.

[10] DIN EN 12390-2 Berichtigung 1: Prüfung von Festbeton - Teil 2: Herstellung und Lagerung von Probekörpern für Festigkeitsprüfungen. Berlin: Beuth 2012.

[11] DIN EN 12390-3: Prüfung von Festbeton - Teil 3: Druckfestigkeit von Probekörpern. Berlin: Beuth 2009.

[12] DIN EN 12390-1: Prüfung von Festbeton - Teil 1: Form, Maße und andere Anforderungen für Probekörper und Formen. Berlin: Beuth 2012.

[13] Zimmer, U. P.; Wöhnl, U.; Breit, W.: Handbuch der Betonprüfung, S. 363-374, 6. Auflage. Düsseldorf: Bau+Technik 2012.

[14] Faust, T.: Leichtbeton im konstruktiven Ingenieurbau. Berlin: Ernst \& Sohn 2003.

[15] Siebert, B.: Bestimmung von Korrelationen zwischen Würfel- und Prismendruckfestigkeit von Vergussmörteln für die Einordnung in Druckfestigkeitsklassen nach DIN EN 206-1/DIN 1045-2. Forschungsbericht DAfStb Projekt-Nr. V450, Bochum: Lehrstuhl für Baustofftechnik der RuhrUniversität Bochum 2006.

[16] DIN EN 12390-5: Prüfung von Festbeton - Teil 5: Biegezugfestigkeit von Probekörpern. Berlin: Beuth 2009.

[17] DIN EN 12390-13: Prüfung von Festbeton - Teil 13: Bestimmung des Elastizitätsmoduls unter Druckbelastung (Sekantenmodul). Berlin: Beuth 2014. 
[18] DIN EN 1352: Bestimmung des statischen Elastizitätsmoduls unter Druckbeanspruchung von dampfgehärtetem Porenbeton und von haufwerksporigem Leichtbeton. Berlin: Beuth 1997.

[19] DIN EN 1992-1-1:2011-01: Eurocode 2: Bemessung und Konstruktion von Stahlbeton- und Spannbetontragwerken - Teil 1-1: Allgemeine Bemessungsregeln und Regeln für den Hochbau+ Nationaler Anhang. Berlin: Beuth 2011 und 2013.

[20] DIN EN 12664: Bestimmung des Wärmedurchlasswiderstandes nach dem Verfahren mit dem Plattengerät und dem Wärmestrommessplatten-Gerät. Berlin: Beuth 2001.

[21] Netzsch Gerätebau GmbH: Wärmeflussmesser - HFM 436 Lambda. Produktbroschüre. Selb: Netzsch 2015.

[22] Zweite Verordnung zur Änderung der Energieeinsparverordnung vom 18.11.2013. In: BGB1. 2013 I S. 3951.

[23] HASIT Fixit 222 Aerogel Hochleistungsdämmputz. Technisches Merkblatt. Freising: 2014.

[24] Zulassung Z-1.6-238: Bewehrungsstab Schöck ComBAR aus glasfaserverstärktem Kunststoff. Berlin: Deutsches Institut für Bautechnik 2014.

[25] RILEM: RC 6 Bond Test for Reinforcement Steel. 2. Pull-out Test. In: RILEM Recommendations for the Testing and Use of Constructions Materials. E \& FN SPON 1994.

[26] Leonhardt, F.: Vorlesung über Massivbau - Teil 1: Grundlagen zur Bemessung im Stahlbetonbau. 3. Auflage, Berlin: Springer-Verlag 1984.

[27] Knippers, J.; Fildhuth, T., Hub, A., Degner, F., Zimmermann, G., Teichmann, T.: Entwicklung eines Leichtbetons mit tragenden und hochdämmenden Eigenschaften als matrixoptimierter und gefügedichter Werkstoff aus zementgebundenem hydrophobierten Aerogelgranulat . Stuttgart: Institut für Tragkonstruktionen und Konstruktives Entwerfen, April 2012.

[28] Fédération Internationale Du Béton (fib): Lightweight Aggregate Concrete - Part 1: Recommended Extensions to Model Code 1990. Lausanne: fib 2000. 


\author{
Autoren: \\ Univ.-Prof. Dr.-Ing. Martina Schnellenbach-Held, massivbau@uni-due.de \\ Dr.-Ing. Torsten Welsch, torsten.welsch@uni-due.de \\ Dipl.-Ing. Silvia Fickler, silvia.fickler@uni-due.de \\ Institut für Massivbau \\ Universität Duisburg-Essen \\ Universitätsstraße 15 \\ D-45141 Essen \\ Dr. rer. nat. Barbara Milow, barbara.milow@dlr.de \\ Prof. Dr. rer. nat. habil. Dr. h.c. Lorenz Ratke, lorenz.ratke@dlr.de \\ Institut für Werkstoff-Forschung \\ Deutsches Zentrum für Luft- und Raumfahrt \\ Linder Höhe \\ D-51147 Köln
}

Bildunterschriften:

Bild 1 Schliffbild eines aus der Referenzmischung hergestellten Probekörpers

Microsection of a specimen made of the reference mixture

Bild 2 Verhältnis zwischen Aerogelanteil und Rohdichte

Relation between amount of aerogel and dry bulk density

Bild 3 Typisches Bruchbild der Aerogelbetonwürfel im Druckfestigkeitsversuch

Typical fracture pattern of the aerogel concrete cubes in the compression tests 
Bild 4 Zusammenhang zwischen Trockenrohdichte und mittlerer Würfeldruckfestigkeit der untersuchten Aerogelbetonproben $\left(* f_{\text {lcm,cube } 150} ; * f_{\text {lcm,prism40 }} \cdot 0,9\right)$

Relation between dry bulk density and compression strength of the tested aerogel concrete specimens $\left(* f_{\text {lcm,cube150 }} ; * f_{\text {lcm,prism } 40} \cdot 0,9\right)$

Bild 5 Zusammenhang zwischen Wärmeleitfähigkeit und mittlerer Würfeldruckfestigkeit der untersuchten Aerogelbetonproben $\left(* f_{\text {lcm,cube } 150} ; * f_{\text {lcm,prism } 40} \cdot 0,9\right)$

Relation between thermal conductivity and compression strength of the tested aerogel concrete specimens $\left(* f_{\mathrm{lcm}, \text { cube } 150} ; * * f_{\mathrm{lcm}, \text { prism40 }} \cdot 0,9\right)$

Bild 6 Schnittfläche des gradierten Aerogelbetonprobekörpers (oben: Wärmedämmschicht; unten: Tragschicht)

Cut surface of graded aerogel concrete (above: heat-insulating layer; below: load bearing layer)

Bild 7 Zusammenhang zwischen Verbundspannung und mittlerer Würfeldruckfestigkeit der untersuchten Aerogelbetonproben bzw. Infraleichtbetonproben $\left(* f_{\mathrm{lcm}, \text { cube } 150} ; * * f_{\mathrm{lcm}, \mathrm{prism} 40} \cdot 0,9\right)$

Relation between bond stress and compression strength of the tested aerogel concrete specimens respectively Infra-Lightweight Concrete $\left(* f_{\text {lcm,cube } 150} ; * f_{\text {lcm,prism } 40} \cdot 0,9\right)$

Tab. 1 Eigenschaften der in [5] - [8] vorgestellten Aerogelbetone

Properties of the Aerogel Concretes presented in [5] - [8]

Tab. 2 Mischungszusammensetzung der Referenzmischung

Composition of the reference mixture

Tab. 3 Druckfestigkeiten, Biegezugfestigkeit und E-Modul verschiedener Aerogelbetonmischungen im Vergleich zu einem Leichtbeton LC8/9 
Compression strength, flexural strength and Young's modulus of different aerogel concrete mixtures compared to a lightweight aggregate concrete LC8/9

Tab. 4 Schallabsorptionskoeffizienten verschiedener Aerogelbetone im Vergleich zu Leicht- und Normalbeton

Sound absorption coefficients of different aerogel concretes compared to a lightweight aggregate concrete and a normal weight concrete 
1

2

3

4

5

6

7

8

9

10

11

12

13

14

15

16

17

18

19

20

21

22

23

24

25

26

27

28

29

30

31

32

33

34

35

36

37

38

39

40

41

42

43

44

45

46

47

48

49

50

51

52

53

54

55

56

57

58

59

60

\begin{tabular}{|c|c|c|c|c|}
\hline Aerogel [Vol.-\%] & $70-50$ & $75-65$ & $60-0$ & $80-0$ \\
\hline$\rho\left[\mathrm{kg} / \mathrm{m}^{3}\right]$ & $580-1.050$ & $500-620$ & $1.000-2.060$ & $760-2.300$ \\
\hline$f_{\mathrm{ccm}}[\mathrm{MPa}]$ & $\left.0,6-1,5^{*}\right)$ & $\left.1,4-2,5^{*}\right)$ & $\left.8,3-62^{*}\right)$ & $\left.0-149^{*}\right)$ \\
\hline$f_{\text {lct,fl }}[\mathrm{MPa}]$ & - & $\left.0,3-0,4^{*}\right)$ & $\left.1,2-7,3^{*}\right)$ & $\left.0,2-19,0^{*}\right)$ \\
\hline$E[\mathrm{MPa}]$ & $52-127$ & $1.100-1.200$ & - & - \\
\hline$\lambda[\mathrm{W} /(\mathrm{mK})]$ & 0,10 & $0,10-0,14$ & $0,26-1,95$ & $0,31-2,30$ \\
\hline
\end{tabular}

*) Prismen mit 40 mm x 40 mm x 160 mm Kantenlänge 


1
1
3
4
5
6
7
8
9
10
11
12
13
14
15
16
17
18
19
20
21
22
23
24
25
26
27
28
29
30
31
32
33
34
35
36
37
38
39
40
41
42
43
44
55
50
56
57
48
59
50
51
53
50

\begin{tabular}{lcc}
\hline & Einheit & Menge \\
\hline CEM I 52,5R & $\mathrm{kg} / \mathrm{m}^{3}$ & 541,0 \\
Aerogelgranulat & Vol.-\% & 61,4 \\
Silicasuspension & Gew.-\%") & 13,0 \\
Fließmittel & Gew..\%") & 3,6 \\
Stabilisierer & Gew.-\%") & 0,5 \\
w/z & - & 0,29 \\
\hline
\end{tabular}

*) bezogen auf den Zementgehalt 


1
2
3
4
5
6
7
8
9
10
11
12
13
14
15
16
17
18
19
20
21
22
23
24
25
26
27
28
29
30
31
32
33
34
35
36
37
38
39
40
41
42
43
40
45
49
50
50
51
53
55
50

\begin{tabular}{|c|c|c|c|c|c|c|c|}
\hline & $\varphi \quad[$ Vol.-\%] & $\rho\left[\mathrm{kg} / \mathrm{m}^{3}\right]$ & $f_{\text {fcm, cube } 150}[\mathrm{MPa}]$ & $f_{c m, c y l}[\mathrm{MPa}]$ & $f_{c t, t,[\mathrm{MPa}]}$ & $f_{\mathrm{ctcm}}[14][\mathrm{MPa}]$ & $E_{1 \mathrm{~cm}}[\mathrm{MPa}]$ \\
\hline M47 & $(\mathrm{LC})$ & 1.360 & 18 & 14,0 & 2,6 & 2,0 & - \\
\hline M48 & 68,3 & 560 & 2,4 & 2,2 & 0,2 & 0,2 & 2008 \\
\hline M50 & 47,0 & 1.180 & 25,5 & 18,2 & 1,8 & 1,4 & 7829 \\
\hline M52 & 60,0 & 840 & 6,7 & 4,9 & 0,4 & 0,3 & 2518 \\
\hline
\end{tabular}




\begin{tabular}{|c|c|c|c|c|c|}
\hline & $\varphi$ & [Vol.-\%] & {$\left[\mathrm{kg} / \mathrm{m}^{3}\right]$} & $f_{\text {cm, cube } 150}[\mathrm{MPa}]$ & $\begin{array}{c}\mu \\
{[1 / \mathrm{cm}]}\end{array}$ \\
\hline (Leichtbeton) & & - & 1.360 & 21,3 & 0,236 \\
\hline M42 & & 68,3 & 540 & 1,7 & 0,234 \\
\hline M43 & & 47 & 1340 & 25,0 & 0,309 \\
\hline M51 & & 60 & 850 & 5,8 & 0,241 \\
\hline Normalbeton & & - & 2.170 & 43,0 & 0,224 \\
\hline
\end{tabular}

13

14

15

16

17

18

19

20

21

22

23

24

25

26

27

28

29

30

31

32

33

34

35

36

37

38

39

40

41

42

43

44

45

46

47

48

49

50

51

52

53

54

55

56

57

58

59

60 


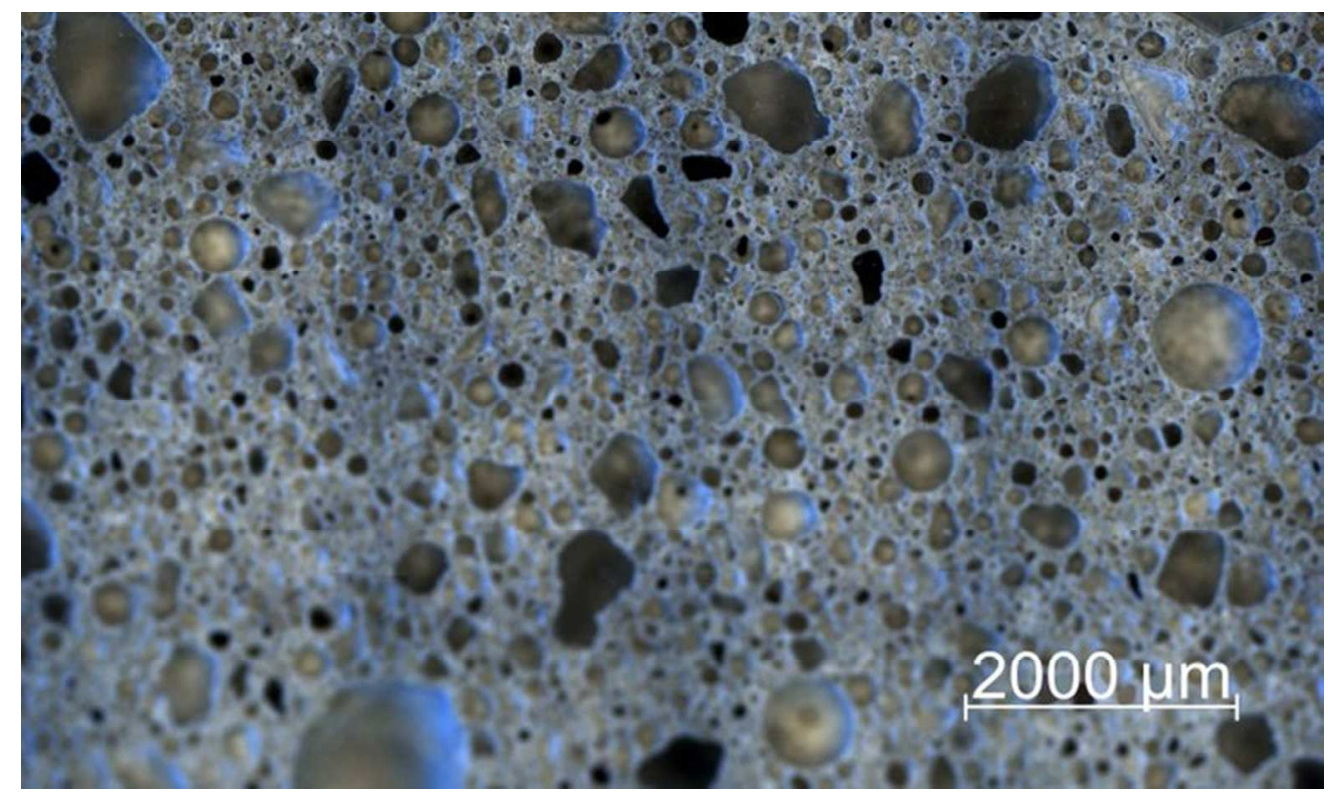

$72 \times 43 \mathrm{~mm}(300 \times 300$ DPI $)$ 


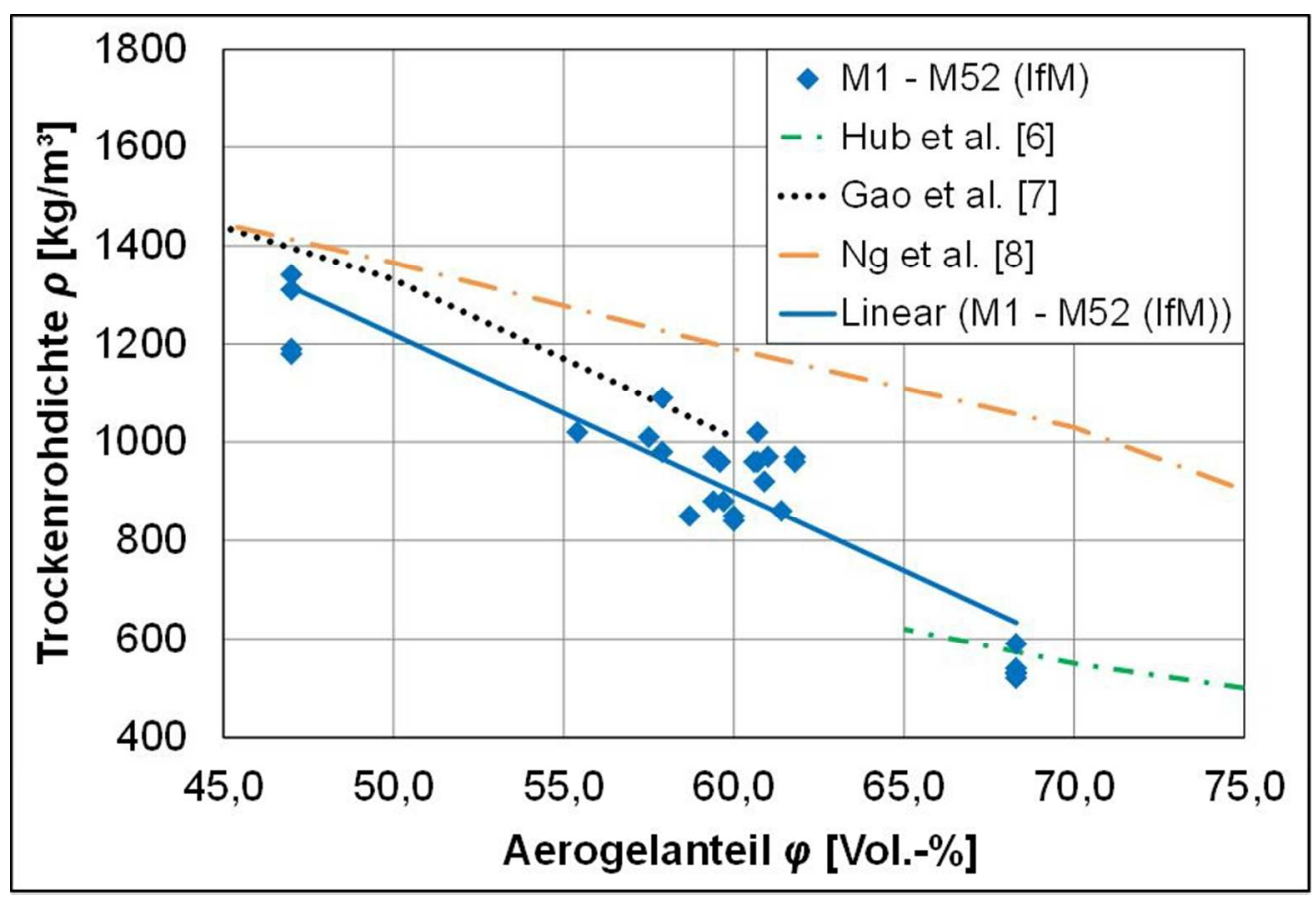

$75 \times 51 \mathrm{~mm}(300 \times 300$ DPI $)$ 


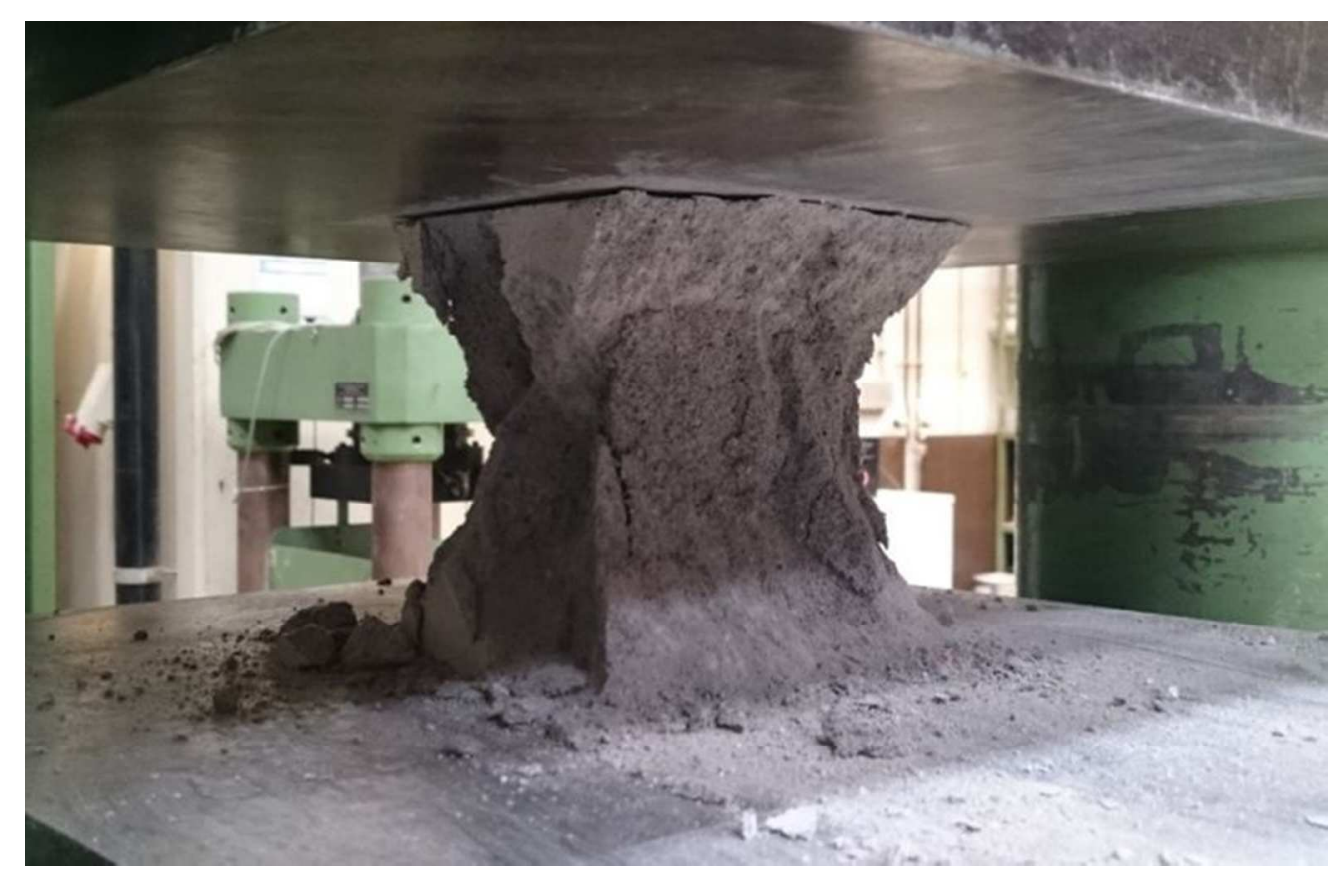

$68 \times 44 \mathrm{~mm}(300 \times 300 \mathrm{DPI})$ 


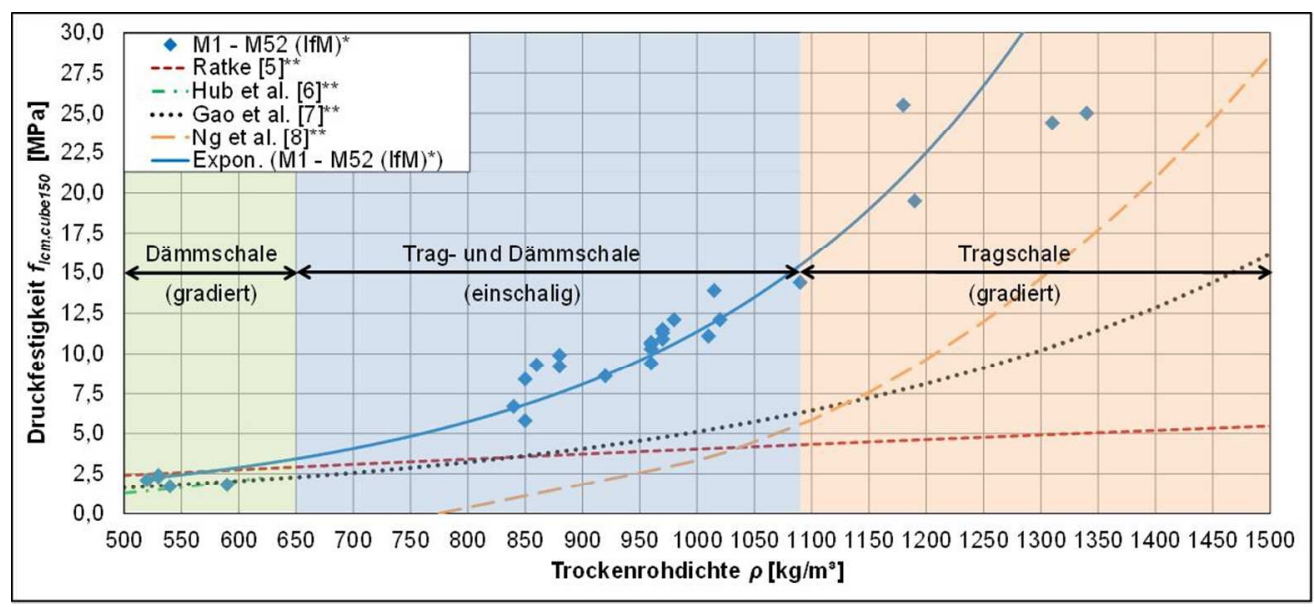

$97 \times 44 \mathrm{~mm}(300 \times 300$ DPI $)$ 


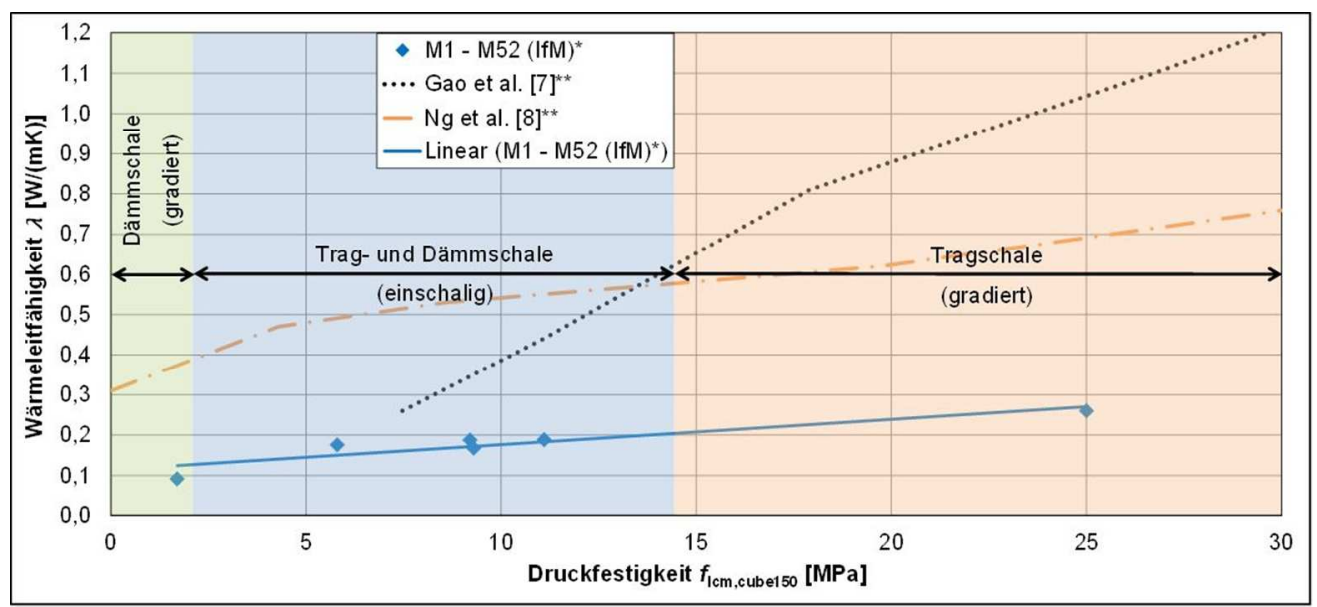

$99 \times 45 \mathrm{~mm}(300 \times 300 \mathrm{DPI})$ 


1
2
3
4
5
6
7
8
9
10
11
12
13
14
15
16
17
18
19
20
21
22
23
24
25
26
27
28
29
30
31
32
33
34
35
36
37
38
39
40
41
42
43
44
45
46
47
48
49
50
51
52
53
54
55
56
57
59
60

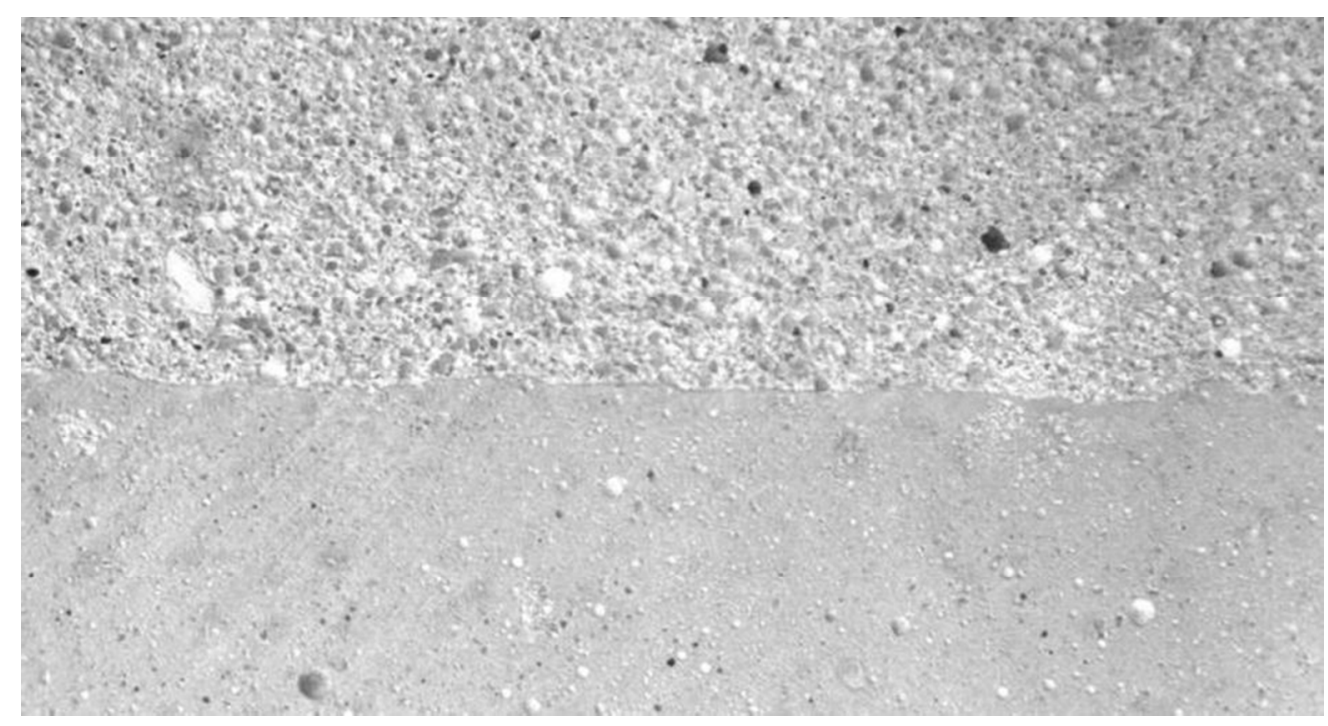

$79 \times 42 \mathrm{~mm}(300 \times 300$ DPI $)$ 


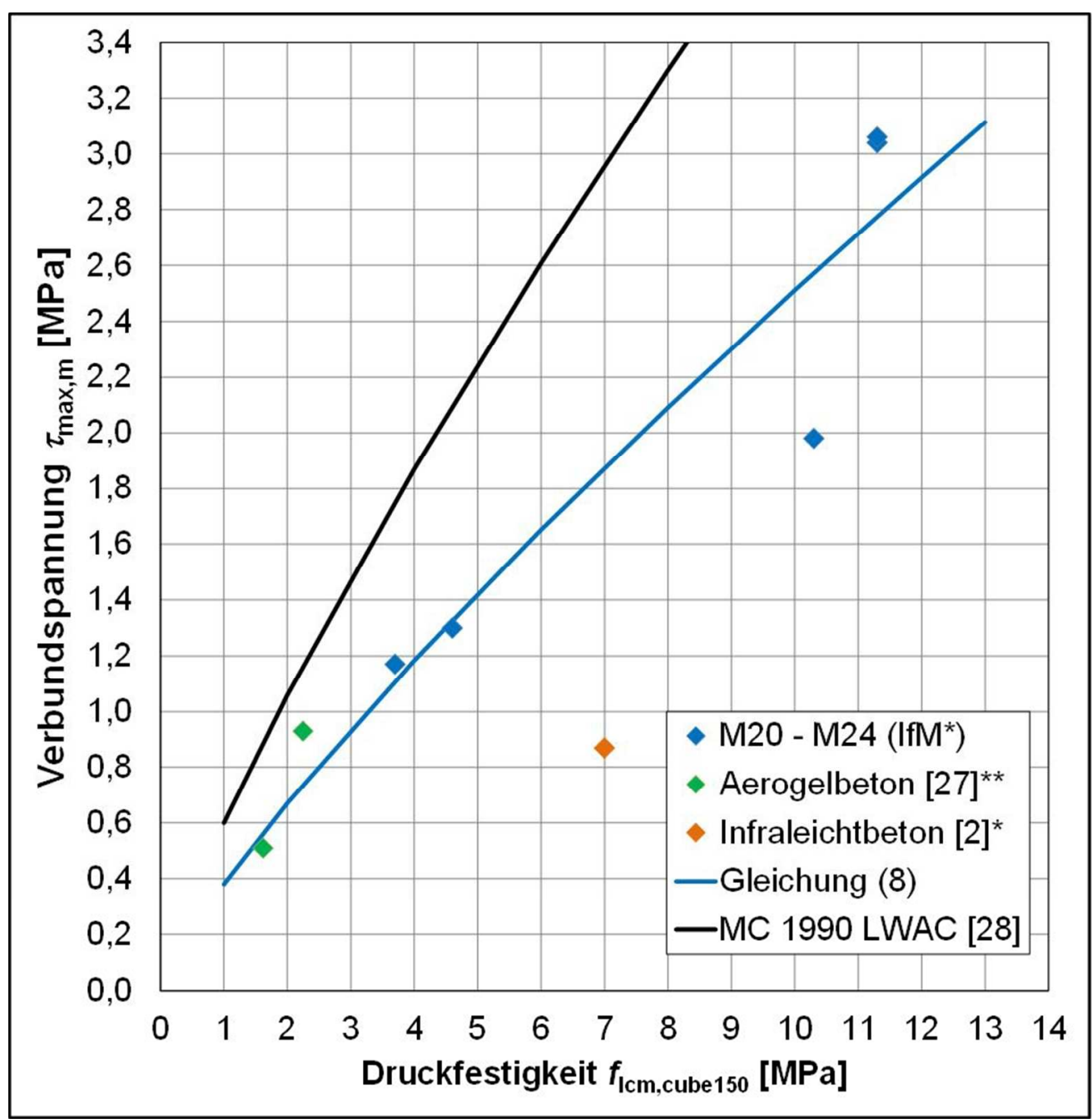

$80 \times 82 \mathrm{~mm}(300 \times 300$ DPI $)$ 


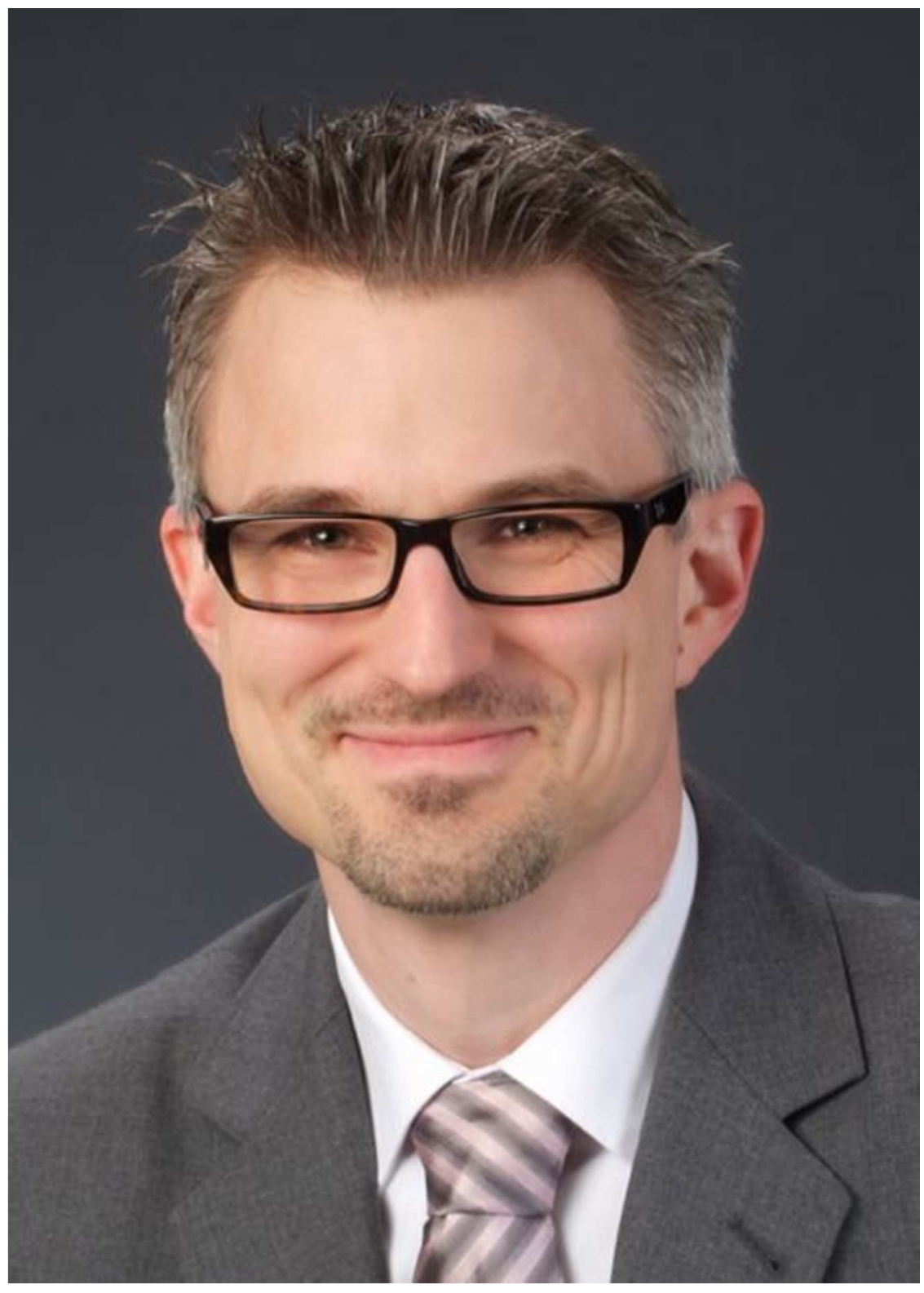

$115 \times 161 \mathrm{~mm}(100 \times 100 \mathrm{DPI})$ 


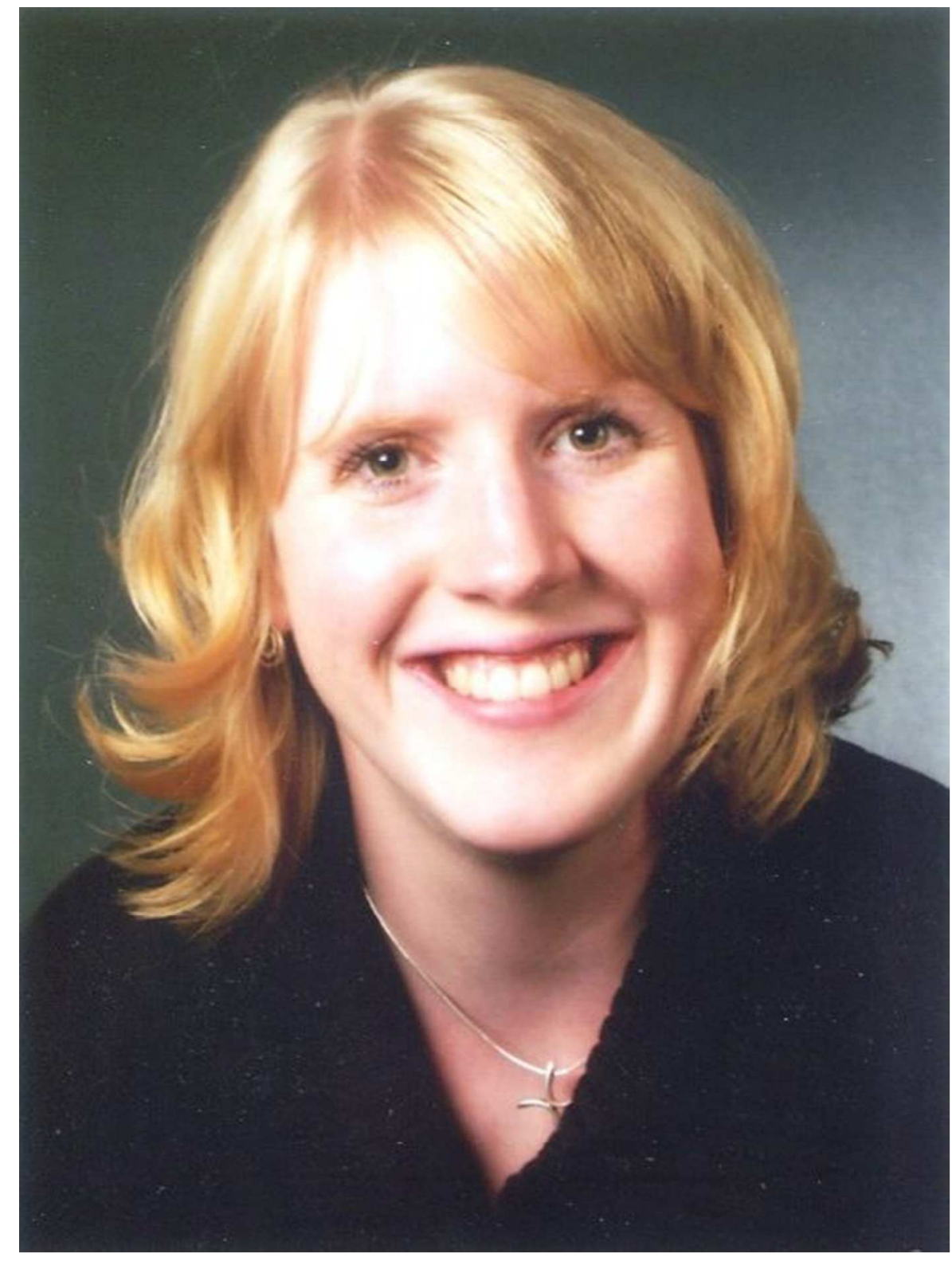

$43 \times 58 \mathrm{~mm}(300 \times 300$ DPI $)$ 


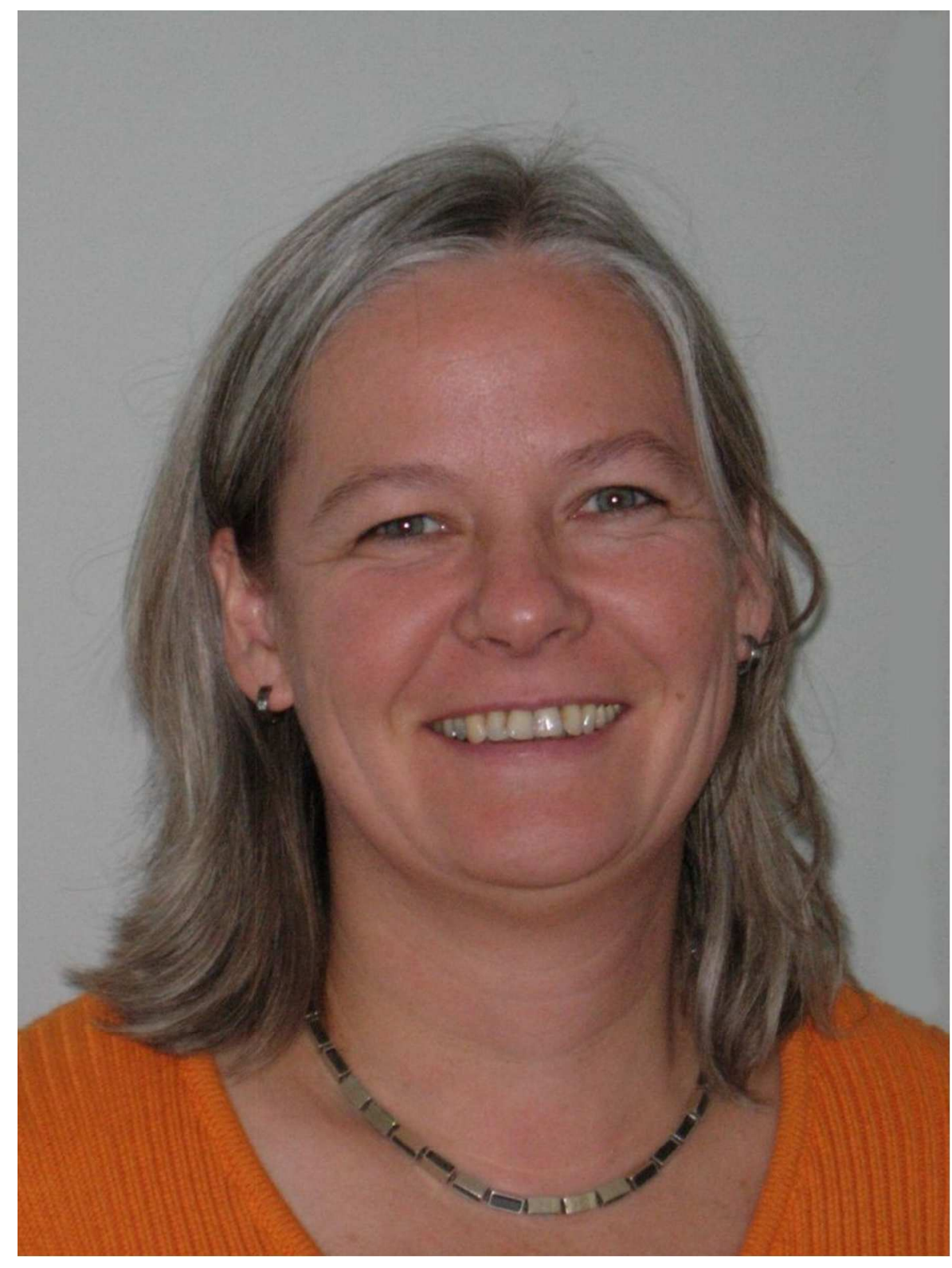

$75 \times 100 \mathrm{~mm}(300 \times 300$ DPI $)$

Wiley-VCH 


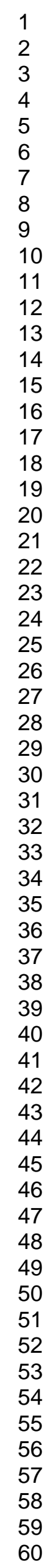

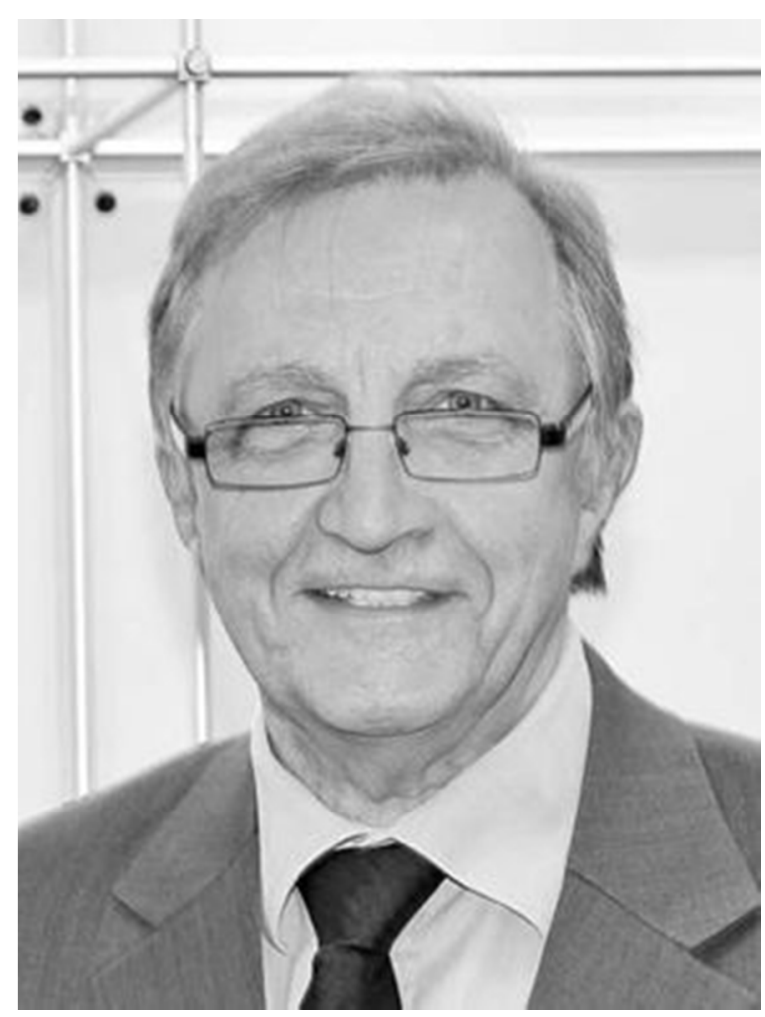

$75 \times 100 \mathrm{~mm}(96 \times 96 \mathrm{DPI})$ 